K.L. Aplin, Composition and measurement of charged atmospheric clusters Accepted for publication in Space Science Reviews, 2008

\title{
Composition and measurement of charged atmospheric clusters
}

\author{
K.L. Aplin \\ Space Science and Technology Department, Rutherford Appleton Laboratory, \\ Chilton, Didcot, Oxon OX11 0QXUK.
}

email k.l.aplin@rl.ac.uk,fax+441235445848

Abstract

Atmospheric charged clusters are formed in a series of rapid chemical reactions after ionisation, leaving a central ion $\mathrm{X}^{+}$or $\mathrm{X}^{-}$clustered with $n$ ligands $(\mathrm{Y})_{n}$. In Solar System tropospheres and stratospheres there are two distinct cluster regimes: the terrestrial planets contain largely hydrated clusters (i.e. $\mathrm{Y}=\mathrm{H}_{2} \mathrm{O}$ ), whereas the gas planets and their moons have organic or nitrogenated cluster species. These classifications are largely based on model predictions, since hardly any measurements are available. The few existing composition measurements are reviewed, including the recent detection of massive charged particles in Titan's upper atmosphere. Technologies for both remote sensing and in situ measurements of atmospheric charged clusters are discussed. Preliminary measurements in the terrestrial atmosphere are presented indicating that ambient charged cluster species interact with downwelling infra-red radiation at $9.15 \mu \mathrm{m}$, even in the presence of cloud. This supports the possibility of future infrared detection of charged clusters.

\section{Keywords}

92.60.Pw Atmospheric electricity, lightning

96.30.Bc Comparative planetology

96.15.Hy Planetary atmospheres

36.40.Wa Charged clusters

94.20.dv Ion chemistry and composition; ionization mechanisms

\section{Introduction}

Ionisation occurs in all planetary atmospheres, principally from cosmic rays (Bazilevskaya et al. 2008). The interaction between an atmospheric molecule and an energetic particle produces an electron and a positive ion. The electron almost immediately attaches to an electronegative molecule (if present) to form a negative ion. After formation of the core ion, there is a rapid $(<1 \mathrm{~ms})$ and complex sequence through which it typically reacts with progressively less abundant trace species through ligand exchange until a stable, terminal ion cluster is produced (Elrod 2003). These "charged clusters", or "cluster-ions", 1 comprise the core ion, hydrogen bonded to one or more polar ligands. Charged cluster production occurs in atmospheres dense and/or cold enough for stable clusters to exist (the collision cross-section is proportional to $p / T^{3 / 2}$ where $p$ is pressure and $T$ temperature). An additional condition for the existence of charged clusters is that the atmosphere must contain suitable polar ligands: either electrophilic species (to form negative

\footnotetext{
${ }^{1}$ Chemically homogeneous clusters can also be referred to as a charged oligomer, e.g. the protonated water dimer.
} 
K.L. Aplin, Composition and measurement of charged atmospheric clusters Accepted for publication in Space Science Reviews, 2008

ions which can subsequently cluster) and/or those with a high proton affinity (to form positive clusters).

Charged clusters interact constantly with each other, aerosol particles and the atmosphere. They gain and lose individual molecules, recombine with oppositely charged particles, and attach to larger particles. On the molecular scale, they attach and recombine to form neutral complexes (Klemperer and Vaida, 2006). The detailed physics of atmospheric ions and their interaction with aerosol particles is discussed in companion papers by Harrison and Tammet (2008) and Tripathi et al. (2008).

There are several motivations to study charged clusters. The direct condensation of vapour onto ions in highly supersaturated conditions has been known since the early days of the cloud chamber. Though the supersaturations needed for this "Wilson" condensation to occur are impossible in the terrestrial atmosphere, there is strong evidence for it in some planetary atmospheres, as will be described in Section 2. Indirect mechanisms have also been postulated for Earth where charged clusters assist nucleation of larger aerosol particles, on which condensation can occur to form cloud droplets (reviewed in e.g. Harrison and Carslaw (2003) and Kazil et al. (2008)). Charged clusters move in electric fields, which can arise either from a global circuit (Aplin et al. 2008) or local generation of charge (Yair 2008). Therefore, in the presence of charge generation mechanisms, charged clusters can represent an important mechanism for the transport of trace chemical species. In some planetary environments charged cluster chemistry could also form trace neutral species (Capone et al. 1979). The radiative effects of charged clusters have been detected in laboratory experiments (Carlon and Harden 1980, Aplin and McPheat 2005), and ionic absorption is likely to have some atmospheric relevance, though the sensitivity of the effect to charged cluster concentration and composition is not yet known. As will be discussed in Section 3 , this technique could be used in the future for remote sensing of charged clusters.

This paper will describe the composition (Section 2) and measurement (Section 3) of atmospheric charged clusters. The discussion focuses on charged clusters in the troposphere and stratosphere, since this is the region where processes discussed in the motivation section above take place.

\section{Composition of charged clusters}

\subsection{Earth}

Typical negative core species in the terrestrial atmosphere are $\mathrm{O}_{2}{ }^{-}, \mathrm{CO}_{3}{ }^{-}$, and $\mathrm{NO}_{3}{ }^{-}$, with positive core species $\mathrm{H}_{3} \mathrm{O}^{+}, \mathrm{NO}^{+}, \mathrm{NO}_{2}{ }^{+}$and $\mathrm{HSO}_{4}{ }^{+}$, and common ligands $\mathrm{H}_{2} \mathrm{O}$ and $\mathrm{NH}_{3}$, where $n \sim 2-10$ (Harrison and Carslaw, 2003). Near the surface, polar organic species act as ligands producing a wide variety of clusters, depending on trace gas concentrations (Elrod 2003; Parts and Luts 2004). Pyridine (an organic heterocyclic molecule with formula $\mathrm{C}_{5} \mathrm{H}_{5} \mathrm{~N}$ ) is a particularly efficient, and therefore common, ligand for positive cluster ions because of its high proton affinity (Elrod 2003), even though it only exists in tiny atmospheric concentrations (Harrison and Tammet 2008). Negative ion species are dominated 
K.L. Aplin, Composition and measurement of charged atmospheric clusters Accepted for publication in Space Science Reviews, 2008

by relatively simple nitrate and sulphate-based clusters, but some organic core species e.g. $\mathrm{C}_{3} \mathrm{H}_{3} \mathrm{O}_{4}{ }^{-}$are also present (Eisele 1989; Eisele et al. 2006). Relatively little is known about ion composition away from the surface, but aircraft mass spectrometer measurements have detected acetone-based cluster ion species e.g. $\mathrm{H}^{+}\left(\mathrm{CH}_{3}\right)_{2} \mathrm{CO}\left(\mathrm{H}_{2} \mathrm{O}\right)_{\mathrm{n}}$ near the tropopause (Möhler et al. 1992). In the upper troposphere there tend to be fewer and less exotic organic charged clusters, and in the lower stratosphere the dominant cluster ions are simpler, such as the protonated hydrate (Smith and Church 1977; Beig and Brasseur 2000). Usually the atomic masses of these ions vary from a few tens to a few hundred atomic mass units (amu), depending on the clustering chemistry which is modulated by e.g. temperature, pressure, and humidity (Harrison and Aplin 2007). Large cluster ions of a few thousand amu have been detected in the upper troposphere using aircraft-borne mass spectrometry (Eichkorn et al. 2002), providing evidence for ion-mediated growth of aerosol particles in cloud-forming regions.

\subsection{Venus}

Borucki et al. (1982) modelled ion clustering reactions in the dense Venusian atmosphere. The clustering processes included were similar to those on Earth, and the terminal clusters may be similar to those in the terrestrial atmosphere, particularly the protonated hydrate, $\mathrm{H}_{3} \mathrm{O}^{+}\left(\mathrm{H}_{2} \mathrm{O}\right)_{n}(n=3-4)$. Other common species are $\mathrm{H}_{3} \mathrm{O}^{+}\left(\mathrm{SO}_{2}\right)$ and $\mathrm{H}_{3} \mathrm{O}\left(\mathrm{H}_{2} \mathrm{O}^{+}\right)\left(\mathrm{SO}_{2}\right)$, with average positive ion mass $\sim 80 \pm 40$ amu. Sulphate species appear to dominate negative ion evolution, with mean mass $\sim 150 \pm 75 \mathrm{amu}$. Aplin (2006) suggested that, based on Magellan data, sulphuric acid supersaturations could be sufficient for direct heterogeneous (Wilson) nucleation of sulphuric acid onto ions in the lower cloud-forming regions, for which the cloud production mechanism is not yet understood.

\subsection{Mars}

Ion formation and evolution in the Martian lower atmosphere was modelled, taking into account measurements of trace gases and water vapour, by MolinaCuberos et al. (2002). Much of the chemistry is similar to the D region of the terrestrial ionosphere at $\sim 75-95 \mathrm{~km}$ (MacGorman and Rust, 1998). The dominant positive species are also similar to those expected on Earth and Venus with the protonated hydrate $(n=2-4)$ most abundant below $70 \mathrm{~km}$. The level of hydration is sensitive to the amount of water vapour present, even though Mars's atmosphere contains an order of magnitude less water vapour than Earth. This is because the proton affinity of water is sufficient for it to attach preferentially to ions, much like pyridine in Earth's atmosphere (Section 2.1). Hydrates such as $\mathrm{CO}_{3}{ }^{-}\left(\mathrm{H}_{2} \mathrm{O}\right)_{\mathrm{n}}$ are the most common negative ions. Nitric acid can occasionally act as a ligand, since its proton affinity is greater than water, though a more common reaction is one in which it acts as a catalyst to produce more protonated hydrates. Despite this complex chemistry, charged clusters play an insignificant role in the proposed Martian global electric circuit, as the expected existence of free electrons will dominate atmospheric conductivity (Aplin 2006).

\subsection{Gas planets: Jupiter, Saturn, Uranus and Neptune}

Ion formation and chemistry at the gas planets was modelled by Capone et al. $(1977,1979)$ and, recently, as part of a study of Jovian aerosol and cloud droplet charging by Whitten et al. (2008). On Jupiter, ions are formed down to $40 \mathrm{~km}$ 
K.L. Aplin, Composition and measurement of charged atmospheric clusters Accepted for publication in Space Science Reviews, 2008

below the visible clouds, below which the atmosphere has absorbed all the ionising radiation from cosmic rays. Terminal positive ions are expected to be $\mathrm{NH}_{4}{ }^{+}$and the clusters $\mathrm{C}_{2} \mathrm{H}_{7}{ }^{+}$and $\mathrm{NH}_{4}{ }^{+}\left(\mathrm{NH}_{3}\right)_{\mathrm{n}}$ (with $2 \geq n \geq 4$ ), which are stable in the relatively cool, yet dense atmosphere. In cloudy regions, electrons formed by ionisation attach rapidly to cloud particles, so the negative ion concentration is negligible. Above and below the cloud layers, negative cluster ions could be significant charge carriers, depending on electrophile concentrations (Whitten et al. 2008). Ionisation could ultimately enhance synthesis of molecules like $\mathrm{C}_{3} \mathrm{H}_{8}$ (propane) and $\mathrm{CH}_{3} \mathrm{NH}_{2}$ (methylamine) in the troposphere. Modelling of ionisation and ion chemistry in the atmospheres of Saturn, Uranus and Neptune predicted that $\mathrm{C}_{2} \mathrm{H}_{9}{ }^{+}$dominated in the lower atmosphere for each planet (Capone et al., 1977, 1979). Negative ions were ignored due to the lack of electrophilic species on Uranus and Neptune, and the rapid reactions expected on Saturn. No further chemical predictions were made, presumably because of the low abundance of reactive trace species known at the time. The models for Saturn, Uranus and Neptune could readily be updated to include more recent measurements of trace species.

\subsection{Titan}

Titan has an extensive nitrogen atmosphere with a surface pressure of $1.5 \mathrm{bar}$, but the chemistry is significantly different to the terrestrial planets. The lack of electrophilic species, such as oxygen, to attach to electrons after ionisation has led to uncertainty over the ratio of free electrons to negative ions in the lower atmosphere (e.g. Borucki et al. 1987; Molina-Cuberos et al. 2001). Since electrons are many orders of magnitude more electrically mobile than molecules, negative atmospheric electrical conductivity and aerosol attachment are both poorly constrained. Recent models have assumed that polyaromatic hydrocarbons (PAHs) are the major electrophilic species, present between $170-350 \mathrm{~km}$, and negative ions are negligible below $100 \mathrm{~km}$ (Whitten et al. 2007). PAHs are expected in Titan's atmosphere from laboratory experiments, and benzene, a chemical precursor to PAHs, was also detected remotely by the Cassini Ion Neutral Mass Spectrometer (Waite et al. 2007). The Cassini Plasma Spectrometer (CAPS) has detected large (a few hundred amu) positive organic ions, and massive (a few thousand amu) negatively charged particles in Titan's upper atmosphere $(\sim 1000 \mathrm{~km})$. The negative particles are thought to play a key role in the formation of the complicated mixture of polymers, called tholins, making up Titan's haze (Coates et al. 2007). This provides further evidence that charge is important for cloud and haze formation in the outer Solar System.

Early results from Huygens have not added much to the earlier modelling work (reviewed in Aplin 2006) on the chemical composition of ions in Titan's lower atmosphere. Techniques developed to enable extraction of ion mobility (related to mass: see Section 3.1 or Harrison and Tammet (2008)) from the Huygens relaxation probe data (Aplin 2005; Owen et al. 2008) could not be used, due to loss of data from the positive channel (Lebreton et al. 2005). In general, the Huygens atmospheric electricity data has been more complicated than expected to analyse due to calibration problems and inconsistencies between the results from the two conductivity instruments (described in more detail in Hamelin et al. 2007). Only relatively preliminary analysis is available, but it is clear from the negative conductivities of $\sim \mathrm{nSm}^{-1}$ reported in Hamelin et al. (2007) that free 
K.L. Aplin, Composition and measurement of charged atmospheric clusters Accepted for publication in Space Science Reviews, 2008

electrons are present, suggesting a lack of electrophilic species. One approach to improve understanding of ion composition would be to combine the ion electrical mobilities predicted in physical models (e.g. Whitten et al. 2007) with up-to-date chemical modelling based on Huygens data e.g. from the Gas Chromatograph Mass Spectrometer, if its detection limit of a mixing ratio of $10^{-8}$ is sufficient (Niemann et al. 2002). It may also be possible that a trace electrophilic species is required below $50 \mathrm{~km}$ to reconcile Huygens measurements and models (Borucki and Whitten 2008), which would presumably lead to negative ion formation.

\subsection{Triton}

Triton's atmospheric ion processes were only studied after Voyager 2 discovered its tenuous atmosphere and thin cloud layer. The atmosphere consists of molecular nitrogen with methane, carbon monoxide and carbon dioxide as the major trace constituents (Delitsky 2006; Delitsky et al. 1990). The stepwise attachment of nitrogen molecules to $\mathrm{CO}^{+}, \mathrm{NO}^{+}$and $\mathrm{C}^{+}$ions produced by cosmic rays or magnetospheric particles is thermodynamically preferred at Triton's low temperatures ( $37 \mathrm{~K}$ at the surface) and forms large nitrogenated clusters e.g. $\mathrm{CO}^{+}\left(\mathrm{N}_{2}\right)_{\mathrm{n}}, \mathrm{NO}^{+}\left(\mathrm{N}_{2}\right)_{\mathrm{n}}$, or $\mathrm{C}^{+}\left(\mathrm{N}_{2}\right)_{\mathrm{n}}$ (where $\left.n \leq 50\right)$. Nitrogen can become highly supersaturated near the tropopause at $9 \mathrm{~km}$ and is expected to condense directly onto the cluster ions. The "Wilson" mechanism of ion-induced nucleation (Aplin 2006 ) is the only mechanism explaining cloud formation on this icy moon. Triton is unique in the Solar System for two reasons, firstly charged clusters appear to be the major process controlling cloud formation and, secondly, the existence of charged clusters is only possible because of the low temperatures favouring nitrogen clustering. Otherwise, the atmosphere is so thin that collision probabilities would be low, making clustering unlikely.

\section{Measurement of charged clusters}

This paper will focus on identification of the composition of atmospheric clusterions rather than bulk electrical properties, although there is some overlap. In the absence of free electrons, charged clusters control air conductivity through their number concentration and electrical mobility (Harrison and Tammet 2008) and this provides a link to studies of the global electric circuit (Aplin et al. 2008). Measurements of the atmospheric electrical properties of charged clusters have already been reviewed by e.g. Aplin (2000), Harrison and Carslaw (2003) and Aplin (2006).

\subsection{In situ measurements}

The terrestrial atmosphere, where charged clusters have been detected for over a hundred years, is the exemplar for in situ measurements (Aplin et al. 2008). In situ charged cluster identification techniques are based on (1) mass spectrometry and (2) electrical mobility.

\subsubsection{Mass spectrometry}

Mass spectrometry of ambient clusters is difficult for several reasons. Firstly the ions can exist in sufficiently small concentrations (mixing ratios of $\sim 10^{-17}$ at the terrestrial surface) that sampling only ambient charged species produces signals that are almost undetectably small. The pressure changes required from ambient pressure down to mass spectrometer operating levels could further reduce the 
K.L. Aplin, Composition and measurement of charged atmospheric clusters Accepted for publication in Space Science Reviews, 2008

signal. Secondly, fragmentation of clusters is common within mass spectrometers, requiring separation of ambient species from those produced in the mass spectrometer. However, mass spectrometry of ambient ions both at the surface and in the free troposphere has been achieved using novel techniques, which are briefly outlined in this section. Tandem mass spectrometry involves stripping cluster ions of their ligands in a dissociation chamber, then selecting a core ("parent") ion using a quadrupole ion trap. This core ion is then fragmented in a buffer gas and the fragments ("daughters") identified with another quadrupole mass filter. The original charged cluster can be identified by comparing the daughter ion spectrum to reference data from artificially produced ions. This technique was used to identify the ion species described in Section 2.1 (Elrod 2003). Similar instruments have been used on planes for measurement of ambient tropospheric ions of up to several thousand amu (Wohlfrom et al. 2000) and identification of "chemiions", charged clusters observed in combustion engine exhausts (Möhler et al. 1992; Kiendler and Arnold 2002).

\subsubsection{Electrical mobility}

Electrical mobility is the speed of an ion in a unit electric field and is related to the ion's mass, charge and the properties of the carrier gas (Harrison and Tammet, 2008). The Rayleigh limit (Strutt, 1882) implies molecular clusters can only carry a single charge, which relates mobility to mass if the carrier gas is known. Ambient ion mobility spectrometers, reviewed by Aplin (2000), separate out ions of different mobilities either by varying the electric field and measuring the change in ion current, or allowing the charged clusters to drift in an electric field and measuring the current as a function of distance. This latter type is known as a drift tube spectrometer, in which ions of different mobility can be identified by their speeds (Nagato and Ogawa, 1998). Drift tube spectrometers take a sample of air which is subsequently analysed, but aspiration ion mobility spectrometers continually blow or suck air through a region (often a cylinder) to which an electric field is applied. The current from ions of different mobilities can be determined by either direct measurement (Tammet 1970; Aplin and Harrison 2001, Fews et al. 2005) or inferred from the rate of voltage relaxation (Aplin 2005). Disadvantages of cluster-ion mobility measurements are that the carrier gas must be well-known, and that the conversion of mobility to mass is non-trivial (Harrison and Tammet 2008)

No in situ extra-terrestrial charged cluster composition measurements have yet been carried out, though it may be possible to infer composition from the results of the Pressure Wave Altimetry experiment on the Huygens probe (Fulchignoni et al. 2002). Miniaturisation of an aspiration-type instrument for possible use in space appears relatively straightforward, but drift tube miniaturisation could be more difficult because decreasing sample volume reduces the ambient ion content (terrestrial instruments often employ an ion source to increase the signal). Drift tubes could also lack spatial resolution if samples were taken, for example, during the descent of a probe.

Mass spectrometry has a well-established space heritage, with numerous instruments flown, often to determine precise isotopic ratios for studies of planetary evolution (e.g. Niemann et al. 2002). Ambient ion measurement would require a broader mass range and lower resolution but with much lower signal strength. There seems no objection in principle to modifying a space-qualified 
K.L. Aplin, Composition and measurement of charged atmospheric clusters Accepted for publication in Space Science Reviews, 2008

quadrupole instrument (e.g. Wright et al. 2007) for in situ detection of atmospheric charged clusters.

\subsection{Remote sensing}

Remote sensing refers here to detection of charged clusters at a distance by a spacecraft in orbit, for which there is not yet a standard technique. The measurement of large ions in Titan's upper atmosphere by the CAPS instrument on Cassini (Section 2.5), an electrostatic analyser intended to detect impacts from aligned fast ion fluxes in Saturn's magnetosphere (Young et al. 2004) were effectively in situ rather than remote detection. CAPS was used in a non-standard way during Titan fly-bys to determine the mass-to-charge ratio of clusters and particles directly impacting the detector. As many of the particles appeared to be massive, and would support multiple charges in the plasma environment of the Titan ionosphere, the CAPS technique used is not adequate to determine composition.

\subsubsection{Laboratory tests of infrared absorption by cluster-ions}

It would be possible to detect atmospheric charged clusters remotely through spectroscopy. Just like neutral molecules and clusters, for which spectroscopic remote sensing is well-established, charged molecular clusters have absorption bands from stretch and bend modes between their hydrogen bonds. Absorption regions, possibly associated with the protonated water oligomer, which is common in the terrestrial atmosphere, and probably also on Mars and Venus, have been identified in the mid-infrared in laboratory experiments (Carlon and Harden 1980, Aplin and McPheat, 2005) (Figure 1). In Aplin and McPheat (2005), ambient cluster-ion concentrations were enhanced using a positive corona source. In this experiment, typical charged cluster concentrations $\sim 8000 \mathrm{~cm}^{-3}$ corresponding to a column concentration in the spectroscopy cell $\sim 10^{13} \mathrm{~m}^{-2}$. As the total column concentration of atmospheric cluster-ions $\sim 10^{14} \mathrm{~m}^{-2}$, the atmospheric absorption signal could be detectable in these bands.

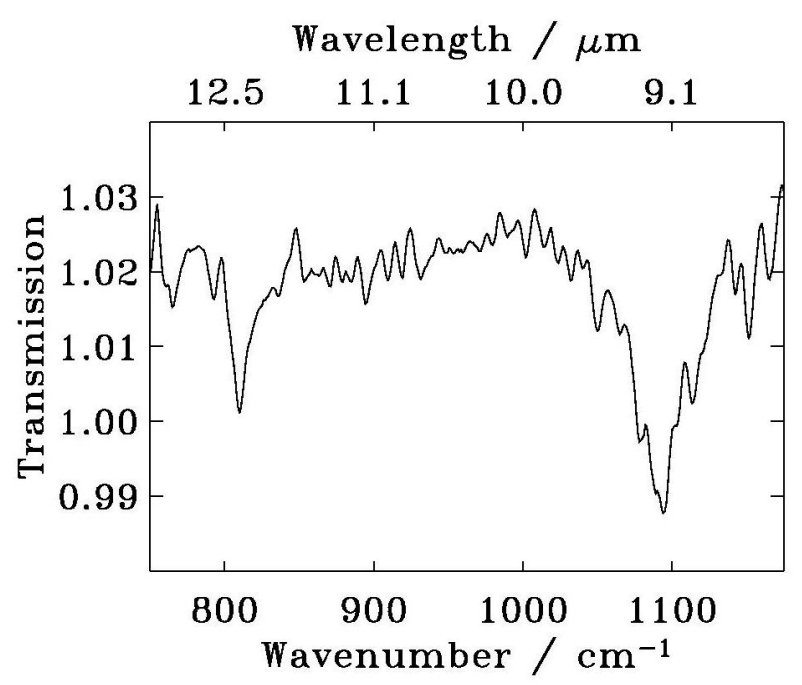

Figure 1 Transmission spectrum of atmospheric charged clusters measured in artificial terrestrial air $\left(T \sim 20^{\circ} \mathrm{C}, p \sim 1000 \mathrm{mbar}\right)$ in a long path cell. The signal shown is the infra-red spectrum at charged cluster column concentrations $\sim 10^{13} \mathrm{~m}^{-2}$, filtered to remove the absorption from neutral water and residual carbon dioxide vapour, and divided by a similarly filtered spectrum obtained at ambient cluster concentrations $\sim 10^{10} \mathrm{~m}^{-2}$. Absorption bands, likely to be from molecular charged clusters can be seen at 12.3 and $9.2 \mu \mathrm{m}\left(815\right.$ and $\left.1090 \mathrm{~cm}^{-1}\right)$. From Aplin and McPheat (2005). 
K.L. Aplin, Composition and measurement of charged atmospheric clusters Accepted for publication in Space Science Reviews, 2008

\subsubsection{Atmospheric instrumentation for measurements of cluster-ion absorption}

To investigate the atmospheric application of this finding, a narrowband filter radiometer system has been developed to measure terrestrial downwelling infrared radiation at a cluster-ion absorption wavelength. The radiometer was deployed at a site where meteorological and electrical measurements are made.

The radiometer was fitted with a narrowband $(9.15 \mu \mathrm{m} \pm 0.75 \%, 5 \%$ bandwidth) interference filter, chosen to match the stronger of the two absorption bands in Figure 1. An auxiliary filter (germanium, outer face coated with diamond-likecarbon, inner face covered with high efficiency anti-reflection coating) was also employed, mounted vertically above the narrowband filter to (1) provide a longwavelength cutoff and (2) to provide robust environmental protection for the narrowband filter. The space between the two filters was purged with nitrogen to remove any residual water vapour, and sealed. A precision, temperature-stable amplifier (x500 gain) (Harrison and Knight 2006) was used to raise the voltage output of the radiometer, and a platinum resistance thermometer attached to the radiometer body.

The air conductivity $\sigma$ is related to the bipolar charged cluster concentration $n$ by.

$$
\sigma \approx n e \bar{\mu}
$$

where $\mu$ is the mean ion mobility at that location (Harrison and Aplin 2007). Conductivity can be determined close to the radiometer from local atmospheric electricity measurements of the Potential Gradient (PG) (Aplin et al. 2008) measured with a field mill, and the conduction current $J_{z}$

$$
\sigma=\frac{J_{z}}{P G}
$$

The parameters measured during the filter radiometer experiment are summarised in Figure 2.

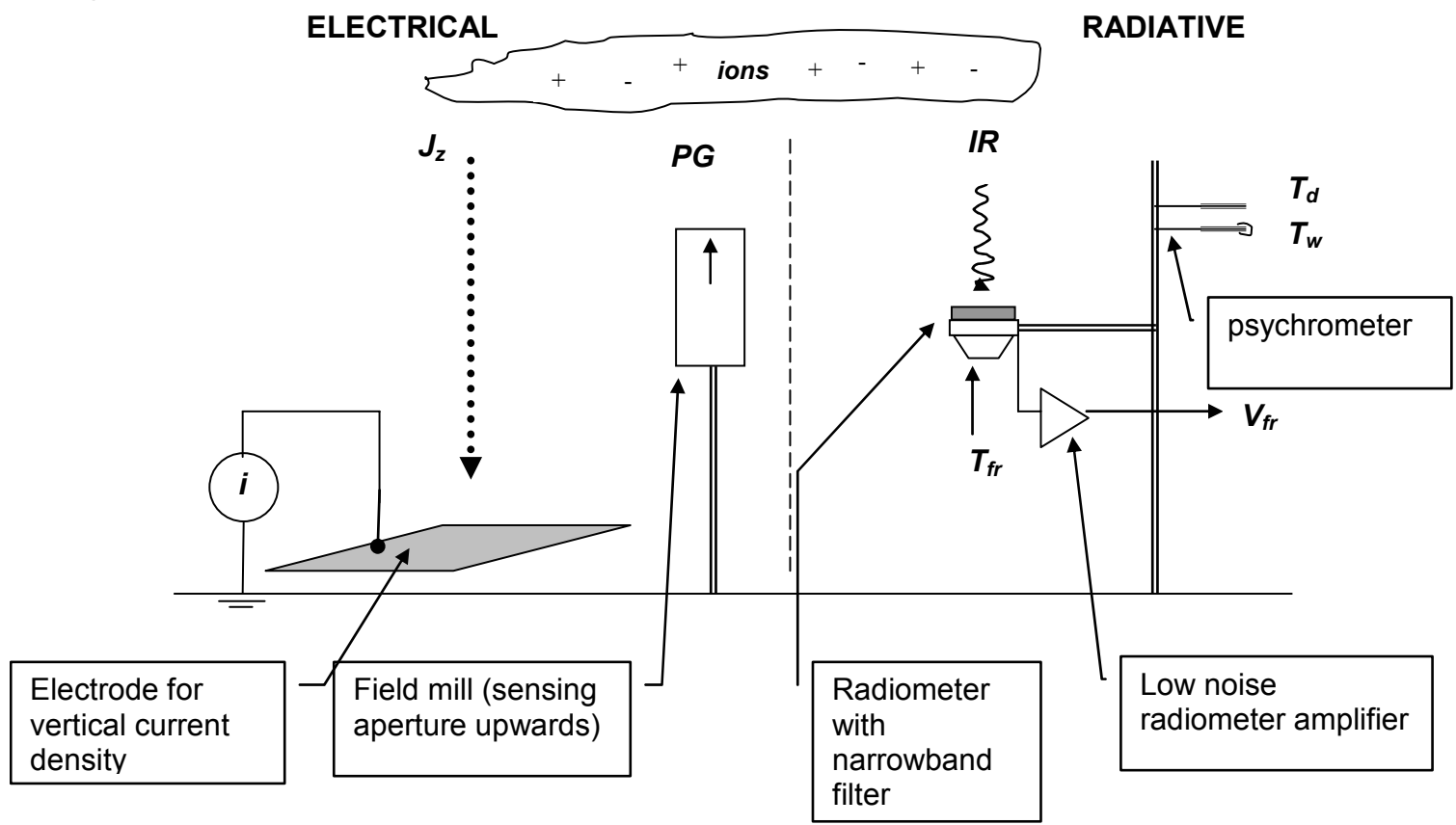

Figure 2 Schematic diagram of co-located electrical and radiative ion measurements. Potential gradient PG is measured using a JCI131 electrostatic field mill. Conduction current $J_{z}$ is detected 
K.L. Aplin, Composition and measurement of charged atmospheric clusters Accepted for publication in Space Science Reviews, 2008

at an insulated metallic electrode (Bennett and Harrison 2008). Dry and wet bulb temperatures $T_{d}$ and $T_{w}$ are used to calculate the water vapour pressure $e$. The filter radiometer body temperature $T_{f r}$ is measured using a platinum resistance thermometer.

\subsubsection{Retrieval of radiometer response to cluster-ion changes}

Preliminary data obtained under clear and foggy conditions are presented here. Nocturnal measurements were chosen to remove solar heating effects. The data were also selected for meteorologically quiescent conditions, particularly low wind speed, to minimise effects from lofting of aerosol particles which could modify the local electrical and radiative environment.

As the ion absorption bands shown in Figure 1 are in the continuum absorption region of the spectrum, the filter radiometer is expected to respond linearly to the column water vapour pressure, represented here by the surface water vapour pressure $e$. Some of the water vapour continuum absorption is thought to be from neutral water vapour clusters, particularly dimers, with concentration approximately proportional to $e^{2}$ (Vaida et al., 2001). The filter radiometer itself will also emit infra-red radiation, proportional to its body temperature $T_{f r}{ }^{4}$. To account for these effects, a multiple linear regression was used to extract the contributions of anomalies in $e, e^{2}, T_{f r}^{4}, T_{f r}$, electrical conductivity $\sigma$, and the interactions between them, on the fluctuations from the median filter radiometer voltage $V_{f r}$. The simplest statistically significant model fitting the data was of the form

$$
V_{f r}=A+B e+C e^{2}+D T+E T_{f r} e+F \sigma
$$

Between the clear and foggy nights, the electrical conductivity coefficient $F$ did not change, within the errors in the regression. This suggests a consistent electrical response of the filter radiometer in the varying conditions. The response of the filter radiometer to cluster-ion concentration changes, determined using (1) and (2), are shown in Figure 3.

(a) Foggy

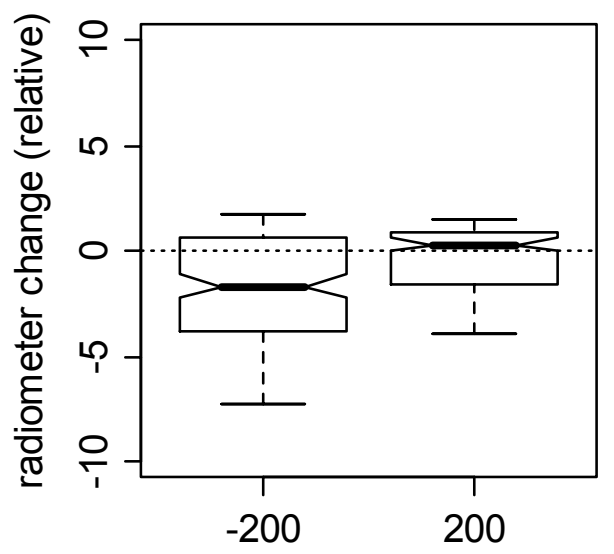

ion concentration change / cc (b) Clear

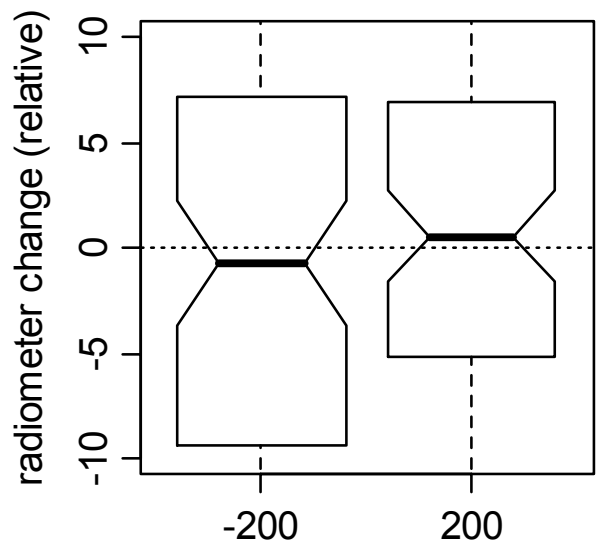

ion concentration change / cc

Figure 3 Relative change in radiometer response, after detrending for vapour pressure, vapour pressure squared and temperature, against the change in ion concentration for (a) foggy (midnight$6 \mathrm{am}$ on $5^{\text {th }}$ November 2007,386 points) and (b) clear conditions (1am-4am on $22^{\text {nd }}$ April 2007, 156 points). 1minute averages are plotted, from $1 \mathrm{~Hz}$ samples, with separated notches indicating $95 \%$ significance. 
K.L. Aplin, Composition and measurement of charged atmospheric clusters Accepted for publication in Space Science Reviews, 2008

Figure 3 indicates that, once other linear effects on the filter radiometer have been removed using equation (3), there is a consistent positive response to changes in ion concentration. This is evidence that the filter radiometer responds to ion changes, and that the signal can be detected in fog, and therefore potentially, through cloud. Further work is required to calculate the expected radiative effect of atmospheric charged water clusters.

Identification of the unique infra-red signals of atmospheric charged clusters even through cloud provides a method for their remote sensing, and therefore also the remote sensing of atmospheric electrical properties (Aplin et al., 2008). The principle could first be demonstrated for the terrestrial atmosphere by comparing satellite observations in the ion absorption bands (appropriate satellite data sets are discussed in e.g. Clerbaux et al. 2003) to in situ ion measurements. For planetary applications, laboratory spectroscopy experiments would be required to generate charged clusters in simulated planetary atmospheres, identify the species and their characteristic absorption bands. It would then be relatively simple to use infra-red spectrometers e.g. the Cassini Composite Infrared Spectrometer (Flasar et al. 2004) to identify atmospheric charged clusters, much like any other trace species.

\section{Discussion}

Charged clusters are common in the Solar System, but measurements of their composition are scarce, even in our own atmosphere. Hydrated clusters are the most abundant species at the terrestrial planets where water vapour is present, because of water's large proton affinity. In the dense atmospheres of Earth and Venus there are approximately equal numbers of positive and negative clusters, but at Mars, free electron stability in the lower atmosphere reduces the number of negative cluster species. At the gas planets and their moons, organic and nitrogenated clusters are expected, with negative clusters relatively rare due to the lack of electrophilic species. Very large charged clusters and charged aerosol particles have been detected directly in Titan's upper atmosphere, though their composition could not be explicitly determined (Coates et al. 2007).

In the terrestrial atmosphere, in situ measurements of the composition can be made through mobility spectrometry or tandem mass spectroscopy, described in section 3.1. Mobility spectrometry instrumentation is smaller, cheaper and can be used for continuous data acquisition without the need for reference laboratory measurements. As mobility links the chemical and physical properties of charged clusters, atmospheric ion mobility spectrometers are versatile and can be used to simultaneously infer atmospheric electrical properties such as air conductivity (Aplin 2000). Existing techniques for mass spectrometry of charged clusters are inherently complicated, with scope for future improvement and simplification, but offer better accuracy in identifying composition.

For planetary applications, mass spectrometry may be more promising for studies of cluster composition, at least in the short to medium term, due to its space heritage. If the instrument is well-characterised before launch, then the need to work off-line generating reference data does not compromise the measurements. Atmospheric ion mobility spectrometers could readily be miniaturised for use in space or other applications. Remote sensing of charged clusters from existing 
K.L. Aplin, Composition and measurement of charged atmospheric clusters Accepted for publication in Space Science Reviews, 2008

satellite spectrometer instruments is potentially a more powerful method with opportunities for wide spatial coverage. Experiments in Earth's atmosphere show that downwelling infra-red radiation in a charged cluster spectral band (Aplin and McPheat 2005) is linked to the cluster-ion concentration, measured independently. This supports the concept of future remote spectroscopic detection of atmospheric charged clusters.

\section{Acknowledgements}

This research was partially funded by the UK Natural Environment Research Council and the Science and Technology Facilities Council. The filter radiometer housing was designed by B. J. Landowski (RAL), and technical support is also acknowledged from A. G. Lomas (Reading University) and W. Robins (RAL). K. A. Nicoll and R.G. Harrison (Reading University) assisted with the filter radiometer measurements.

\section{References}

K.L. Aplin, Instrumentation for atmospheric ion measurements, PhD Thesis, The University of Reading, UK (2000)

K.L. Aplin, Surv. Geophys., 27, 63-108 doi: 10.1007/s10712-005-0642-9 (2006)

K.L. Aplin, Rev. Sci. Instrum, 76, 104501 doi: 10.1063/1.2069744 (2005)

K.L. Aplin, R.G. Harrison, Rev. Sci. Instrum, 72, 8 3467-3469 (2001)

K.L. Aplin, R.G. Harrison, M.J. Rycroft, this issue (2008)

K.L. Aplin, R.A. McPheat, J. Atmos. Sol-Terr Phys, 67, 8-9, 775-783 doi:10.1016/j.jastp.2005.01.007 (2005)

G. A. Bazilevkaya, I.G. Usoskin, E. Flückiger et al., this issue (2008)

A.J. Bennett, R.G. Harrison, J. Atmos. Solar-Terr. Phys. doi: 10.1016/j.jastp.2008.04.014 (in press, 2008)

G. Beig, G.P. Brasseur, J. Geophys. Res. 105, 22671-22684 (2000)

W.J. Borucki, Z. Levin, R.C. Whitten et al., Icarus, 51, 302-321 (1982)

W.J. Borucki, Z. Levin, R.C. Whitten et al., Icarus, 72, 604-622 (1987)

W.J. Borucki, R.C. Whitten, Planet. Space Sci., 56, 19-26, doi:10.1016/j.pss.2007.03.013 (2008)

L.A. Capone, J. Dubach, R.C. Whitten et al. Icarus 39, 433-449 (1979)

L.A. Capone, R.C. Whitten, S.S. Prasad et al. Ap. J. 215, 977-983 (1977)

H. R. Carlon, C. S. Harden, App. Optics 19, 11, 1776-1786 (1980)

C. Clerbaux, J. Hadji-Lazaro, S. Turquety et al., Atmos. Chem. Phys., 3, 1495-1508 (2003)

A. J. Coates, F. J. Crary, G. R. Lewis, et al., Geophys. Res. Lett. 34, L22103, doi:10.1029/2007GL030978 (2007)

M.L. Delitsky, R.P. Turco, M.Z. Jacobson, Geophys. Res. Lett. 17, 10, 1725-1728 (1990)

M.L. Delitsky, Bull. Amer. Astron. Soc., 38, 519 (2006)

S. Eichkorn, S. Wilhelm, H. Aufmhoff et al., Geophys. Res. Lett., 29, 14, 1698, doi:10.1029/2002GL015044 (2002)

F.L. Eisele, J. Geophys. Res., 94, 2183-2196 (1989)

F.L. Eisele, E. R. Lovejoy, E. Kosciuch et al., J. Geophys. Res., 111, D04305, doi:10.1029/2005JD006568 (2006)

M.J. Elrod, Int. J. Mass Spec., 228, 91-105 (2003)

A.P. Fews, N.K. Holden, P.A. Keitch et al., Atmos. Res. 76, 1-4, 29-48 (2005)

F. M. Flasar, V.G. Kunde, M.M. Abbas et al., Space Sci, Rev. 115, 1-4, 169-297 (2004)

M. Fulchignoni, F. Ferri, F. Angrilli et al, Space Sci. Rev. 104, 1-4, 395-431, doi:

10.1023/A:1023688607077 (2002)

M. Hamelin, C. Beghin, R. Grard et al., Planet. Space Sci. 55, 1964-1977,

doi:10.1016/j.pss.2007.04.008 (2007)

R.G. Harrison, K.L. Aplin, Atmos. Res., 85, 199-208, doi:10.1016/j.atmosres.2006.12.006 (2007)

R.G. Harrison, K. S. Carslaw, Rev. Geophys., 41, doi: 10.1029/2002RG000114 (2003)

R.G. Harrison, J.R. Knight, Rev. Sci. Instrum. 77, 116105 doi: 10.1063/1.2370752 (2006)

R.G. Harrison, H. Tammet, this issue (2008)

J. Kazil, R.G. Harrison, E. Lovejoy et al., this issue (2008)

A. Kiendler, F. Arnold, Atmos. Env. 36, 1757-1761 (2002)

W. Klemperer, V. Vaida, Proc. Nat. Acad. Sci., 103, 28, 10584-10588,

doi:10.1073/pnas.0508231103 (2006) 
K.L. Aplin, Composition and measurement of charged atmospheric clusters Accepted for publication in Space Science Reviews, 2008

J.P. Lebreton, O. Witasse, C. Sollazzo et al., Nature 438, 758-764 doi:10.1038/nature04347 (2005)

O. Möhler, T. Reiner, F. Arnold, Rev. Sci. Instrum. 64, 5, 1199-1207 (1993)

D.R. MacGorman, W.D. Rust, The electrical nature of storms (Oxford University Press, Oxford, 1998), pp 25-31

G.J. Molina-Cuberos, J.J López-Moreno, R. Rodrigo et al., Adv. Space Res. 28, 10, 1511-1516 (2001)

G.J. Molina-Cuberos, H. Lichtenegger, K. Schwingenschuh et al., J. Geophys. Res. 107, E5, doi: 10.1029/2000JE001447 (2002)

K. Nagato, T. Ogawa, J. Geophys. Res, 103, 13917-13925 (1998)

H.B. Niemann, S.K. Atreya, S.J. Bauer et al., Space Sci. Rev., 104, 1-4, 553-591, doi:10.1023/A:1023680305259 (2002)

N. R. Owen, K.L. Aplin, P.A. Stevens, J. Phys. Conf. Ser. (in press, 2008)

T.E. Parts, A. Luts, Atmos. Env. 38, 1283-1289, doi:10.1016/j.atmosenv.2003.11.030 (2004)

D. Smith, M. J. Church, Planet. Space Sci. 25, 5, 433-439 doi:10.1016/0032-633(77)90075-7 (1977)

J. W. Strutt (Lord Rayleigh), Phil. Mag. 14, 184-186 (1882)

$\mathrm{H}$. Tammet, The aspiration method for the determination of atmospheric ion spectra (Israel program for scientific translations, Jerusalem, 1970)

S.N. Tripathi, M. Michael, R.G. Harrison, this issue (2008)

V. Vaida, J.S. Daniel, H.G. Kjaergaard et al., Q. J. Roy. Met. Soc., 127, 1627-1643 (2001)

J. H. Waite, D. T. Young, T. E. Cravens et al., Science 316, 5826, 870-875 doi:

10.1126/science.1139727 (2007)

R.C. Whitten, W.J. Borucki, S.N. Tripathi, J. Geophys. Res. 112, E04001, doi:

10.1029/2006JE002788 (2007)

R.C. Whitten, W.J. Borucki, K. O'Brien et al., J. Geophys. Res. 113, E04001, doi:

10.1029/2007JE002975 (2008)

K. H. Wohlfrom, S. Eichkorn, F. Arnold et al., Geophys. Res. Lett. 27, 23, 3853-3860 (2000)

I.P. Wright, S. J. Barber, G.H. Morgan et al., Space Sci. Rev, 128, 1-4, doi: 10.1007/s11214-0069001-5 (2007)

Y. Yair, Space Sci. Rev, this issue (2008)

D.T. Young, J.J. Berthelier, M. Blanc et al., Space Sci. Rev., 114, 1-4, 1-112 doi 10.1007/s11214004-1406-4 (2004) 